\title{
Avaliação da Medida do Comprimento do Colo e da Ausência do Eco Glandular Endocervical para Predição do Parto Pré-Termo
}

\author{
Evaluation of Risk for Preterm Delivery by Measurement of Uterine Cervix and Cervical Gland Area
}

Claudio Rodrigues Pires, Antonio Fernandes Moron, Rosiane Mattar, Luiz Kulay Júnior

\section{RESUMO}

Objetivo: verificar a prevalência do sinal eco glandular endocervical (EGE) e o comprimento cervical menor ou igual a $20 \mathrm{~mm}$ em gestantes entre a $21^{a}$ e a $24^{a}$ semana e comparar estes sinais ecográficos como fatores indicadores de parto pré-termo espontâneo.

Métodos: estudo prospectivo transversal no qual foram incluídas 361 gestantes da população geral, para realização de exame ultra-sonografico em idade gestacional entre a $21^{a}$ a $24^{a}$ semana. Os critérios de exclusão do estudo foram malformações müllerianas, gestações múltiplas, malformações fetais, óbito fetal, alterações da quantidade de líquido amniótico, placenta com inserção segmentar, antecedentes de cirurgia no colo uterino (conização, amputação, cerclagem) e procedimentos cirúrgicos durante a gestação. Após a realização do exame ultra-sonográfico obstétrico morfológico efetuado por via abdominal, seguiu-se o exame ecográfico por via vaginal para observação de uma faixa hipoecóica ou hiperecóica adjacente ao canal endocervical correpondente às glândulas do epitélio endocervical (EGE) $e$ mensuração do comprimento cervical. As variáveis qualitativas são representadas por freqüência absoluta e relativa, ao passo que as variáveis quantitativas, por média, desviopadrão, mediana e valores mínimo e máximo. A associação entre as variáveis qualitativas foi avaliada pelo teste $\chi^{2}$ ou teste exato de Fisher. Para cada variável estudada, foi calculado o risco relativo seguido do intervalo com 95\% de confiança. A técnica de análise de regressão logistica univariada foi utilizada para verificar, entre as variáveis estudadas, quais foram indicativas de parto pré-termo espontâneo. O nivel de significância adotado foi de 95\% (alfa $=5 \%$ ) e descritivos (p) iguais ou inferiores a 0,05 foram considerados significantes.

Resultados: a incidência do parto pré-termo espontâneo foi de 5,0\%. O comprimento do colo uterino revelou-se igual ou inferior a $20 \mathrm{~mm}$ em 3,3\% da população estudada e em 27,8\% das pacientes que apresentaram parto pré-termo espontâneo. A ausência do EGE foi detectada em 2,8\% das pacientes estudadas e em 44,4\% das pacientes que evoluíram para parto pré-termo espontâneo. A associação entre ausência do EGE e presença do colo curto revelou-se estatisticamente significante $(p<0,001)$. A ausência do EGE teve forte associação com parto pré-termo espontâneo e risco relativo de 28,57, com intervalo de confiança (IC 95\%) 14,4056,68 . A medida do comprimento cervical inferior a $20 \mathrm{~mm}$ também apresentou associação com parto pré-termo espontâneo ( $p<0,001$ ), com risco relativo de 11,27 e IC 95\% de 4,79-26,53. Conclusão: a não visualização do EGE endocervical constitui parâmetro morfológico ultrasonográfico novo e útil na predição do parto pré-termo espontâneo nas gestações únicas da população geral. Os resultados deste trabalho indicam uma tendência clara da marcante importância da ausência do EGE como indicador do risco para parto pré-termo espontâneo, a ser confirmada em pesquisas multicêntricas futuras.

PALAVRAS-CHAVE: Colo uterino. Parto pré-termo. Comprimento do colo. Eco glandular endocervical. 
Introdução

A prematuridade é, nos dias atuais, um dos principais problemas de saúde no periodo perinatal, constituindo a principal causa de mortalidade neonatal ${ }^{1,2}$. O parto pré-termo (PPT), ou seja, o que ocorre antes da $37^{\mathrm{a}}$ semana completa, constitui importante determinante da morbidade neonatal e infantil, incluindo alterações do desenvolvimento neuro-motor, doenças respiratórias crônicas, predisposição para processos infecciosos e distúrbios oftalmológicos ${ }^{3}$.

Muitas publicações destacam métodos clínicos, marcadores biológicos, bioquímicos e ultrasonográficos que, aplicados isolada ou combinadamente, buscam predizer o PPT espontâneo ${ }^{1,4-6}$. O exame ultra-sonográfico transvaginal (USTV) é o método mais eficaz para o estudo do colo uterino durante a gravidez e permite avaliar a morfologia e a biometria cervical com alto grau de confiabilidade ${ }^{7}$.

Há mais de uma década, acumulam-se os trabalhos estabelecendo as medidas cervicais indicativas do PPT, sendo o comprimento cervical o principal parâmetro utilizado. A maioria destas publicações apresenta uma conclusão comum: a de que, quanto menor o comprimento cervical, maior o risco para PPT espontâneo ${ }^{8-15}$. Assim, embora diferindo muito quanto aos métodos e populações estudadas, estes resultados foram, aos poucos, estabelecendo medidas de referência que podem ser utilizadas como indicativas ${ }^{7}$. Entretanto, o limite do comprimento do colo uterino representativo de risco para PPT ainda é motivo de controvérsia na literatura. Diversos estudos têm indicado comprimentos que variam de 15 a $35 \mathrm{~mm}^{2,9-12,15-17}$.

Nos últimos anos, três publicações de um mesmo grupo de pesquisadores ressaltaram a importância de um marcador US morfológico do colo uterino passível de visualização pela US, a denominada cervical gland area, ou área das glândulas endocervicais. Quando não detectado este sinal, parece relacionar-se com processo de maturação cervical $^{18-20}$. Sekiya ${ }^{18}$, em 1998, coordenou estudo que revelou pela primeira vez que a não-identificação da área glandular cervical à US poderia corresponder ao início do processo de maturação do colo uterino. O mesmo grupo, no ano de 2002, observou relação entre as taxas de detecção das glândulas endocervicais, comprimento cervical, índice de

Universidade Federal de São Paulo - Escola Paulista de Medicina - Departamento de Obstetrícia - Disciplina de Medicina Fetal Correspondência:

Cláudio Rodrigues Pires

Rua Capitão Garcindo 145 - Pacaembu

01250-010 - São Paulo - SP

e-mail: crpires@uol.com.br maturação cervical e resultado da gestação. Segundo este estudo, a não visualização da área das glândulas endocervicais poderia ser considerada como indicador de falha dos resultados no processo de inibição do trabalho de parto pré-termo ${ }^{19}$. No ano seguinte, Fukami et al. ${ }^{20}$, também do mesmo departamento, estudaram a área das glândulas endocervicais em pacientes com idade gestacional entre a $16^{\mathrm{a}}$ e a $19^{\mathrm{a}}$ semana de gestação por meio da avaliação US-TV. Nesta ocasião, os autores compararam a não-visualização da cervical gland area com o comprimento do canal endocervical e observaram maior sensibilidade e significativa elevação do valor preditivo positivo para PPT anterior à $32^{\mathrm{a}}$ semana de gestação quando comparado ao marcador biométrico, o comprimento cervical.

Assim, tivemos a intenção de avaliar morfológica e biometricamante o colo uterino, por ocasião do exame US obstétrico morfológico do segundo trimestre, idade gestacional na qual os estudos ecográficos morfológicos do colo uterino são escassos. O presente estudo teve por objetivo verificar a prevalência do sinal cervical gland area e comprimento cervical inferior a $20 \mathrm{~mm}$ em gestantes entre a $21^{\text {a }}$ e a $24^{\text {a }}$ semana e avaliar como valor preditivo de risco para PPT espontâneo.

\section{Pacientes e Métodos}

O presente estudo, analisado e aprovado pelo Comitê de Ética em Pesquisa da UNIFESP e do Hospital São Paulo, foi desenvolvido de forma prospectiva, transversal e incluiu uma população de mulheres grávidas submetidas a exame US obstétrico de rotina no primeiro trimestre encaminhadas ao Centro de Treinamento em Ultra-sonografia de São Paulo. Estas pacientes eram oriundas da rede do Sistema Único de Saúde (SUS) e de serviços universitários do município de São Paulo.

Os critérios de inclusão foram grávidas da população geral, com feto único e vivo, entre a $21^{\text {a }}$ e a $24^{\text {a }}$ semana de gestação. Os critérios de exclusão do estudo foram pacientes portadoras de malformações müllerianas, gestações múltiplas, malformações fetais, óbito fetal, alterações da quantidade de liquido amniótico, placenta com inserção baixa, antecedentes de cirurgia no colo uterino (conização, amputação, cerclagem), procedimentos cirúrgicos durante a gestação, PPT induzido na presente gestação e não-comparecimento na data agendada para o exame US.

A avaliação US obstétrica por via abdominal até a $14^{a}$ semana de gestação em 685 mulheres permitiu determinar a idade gestacional de forma 
acurada, a qual foi utilizada como parâmetro básico para estimativa da idade da gestação por ocasião do exame morfológico do segundo trimestre e no dia do parto. Na primeira avaliação uma ficha cadastral foi preenchida com dados pessoais como nome completo, endereço e telefone, além de informações sobre os antecedentes pessoais, obstétricos e familiares. Na mesma ocasião foi proferida uma explanação sobre os exames a serem realizados e a finalidade deste estudo e as pacientes que concordaram em participar do mesmo leram e assinaram um termo de informação e consentimento redigido segundo as normas do Comitê de Ética em Pesquisa da UNIFESP/Hospital São Paulo. As mesmas gestantes eram convidadas a retornar na $21^{\text {a }}$ e na $24^{a}$ semana gestacionais para US morfológica fetal, conforme rotina do serviço. Em razão do não-comparecimento na data agendada ou em decorrência dos critérios de exclusão, 425 pacientes foram submetidas ao estudo US-TV do colo uterino após avaliação morfológica fetal.

Quarenta e duas pacientes foram retiradas da análise final por apresentarem algum critério de exclusão ou por perda de seguimento impossibilitando a obtenção de dados do parto. Foram identificadas 40 pacientes com PPT, sendo 22 induzidos, que foram excluídas, e 18 espontâneos. Desta forma, a amostragem final totalizou 361 gestantes.

A média de idade das gestantes era de 25 anos (DP \pm 6 anos). Quanto aos antecedentes obstétricos, o número de gestações variou entre uma e nove gestações, com $145(40,2 \%)$ mulheres primigestas, $102(28,3 \%)$ secundigestas, 65 $(18,0 \%)$ tercigestas e $49(13,6 \%)$ com quatro ou mais gestações. $\mathrm{O}$ número de partos anteriores das pacientes da amostra variou entre zero e cinco partos, com $170(47,1 \%)$ nuliparas, $103(28,5 \%)$ com um parto e $88(24,4 \%)$ com dois ou mais partos. O número de abortamentos variou entre zero e quatro, com $289(80,1 \%)$ sem nenhum aborto, $52(14,4 \%)$ com um aborto e $20(5,5 \%)$ com dois ou mais abortos anteriores.

Apenas um médico, o autor deste artigo, com treinamento especializado em US, realizou todas as medidas e avaliações US morfológicas cervicais deste estudo tendo obedecido os passos descritos a seguir. A avaliação US-TV foi efetuada com equipamentos de US de alta resolução, com transdutores endocavitários de $5,0 \mathrm{MHz}$ a $6,5 \mathrm{MHz}$ e ângulo de visão igual ou superior a $120^{\circ}$, apropriados para tal procedimento. Um preservativo sem lubrificante recoberto com gel de transmissão ultra-sônica revestiu o transdutor, de acordo com a técnica de exame US-TV.

Antes do exame US, as pacientes foram orientadas para proceder ao esvaziamento vesical completo. Posteriormente as gestantes foram colocadas em posição ginecológica, em decúbito dorsal, com os membros inferiores abduzidos, dispostos de forma simétrica, permitindo a movimentação livre do transdutor.

Após a identificação do orificio interno do colo, o transdutor foi movimentado até a obtenção da imagem de toda extensão do canal cervical, seguindose a pesquisa da área das glândulas endocervicais e do orificio cervical externo. Uma imagem adequada foi definida como a visualização nítida dos orifícios interno e externo do colo e de toda extensão do canal cervical. A distância entre os dois pontos foi denominada comprimento cervical. Esta medida foi repetida por três vezes durante um período de pelo menos cinco minutos e foi considerada para análise a menor medida obtida neste período.

A "área das glândulas endocervicais" foi renomeada nesta publicação de "eco glandular endocervical" (EGE), definida como zona hiperecóica ou hipoecóica em torno do canal endocervical (Figura 1), a qual corresponde à área histológica das glândulas ${ }^{18-20}$. Se o EGE em torno do canal endocervical não fosse visualizado, era definido como ausente (Figura 2).

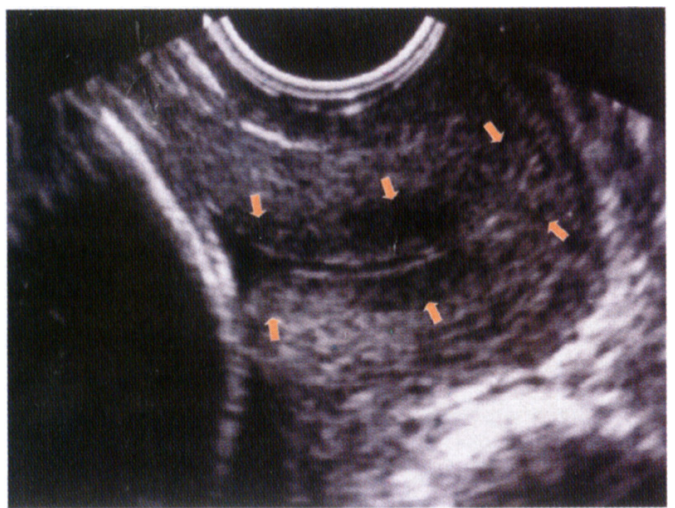

Figura 1 - Exame ultra-sonográfico evidenciando colo uterino em corte longitudinal. As setas apontam a transição entre o eco glandular endocervical (EGE) (aspecto hipoecóico) e o estroma cervical. Observa-se o canal endocervical com pequena quantidade de muco (anecóico).

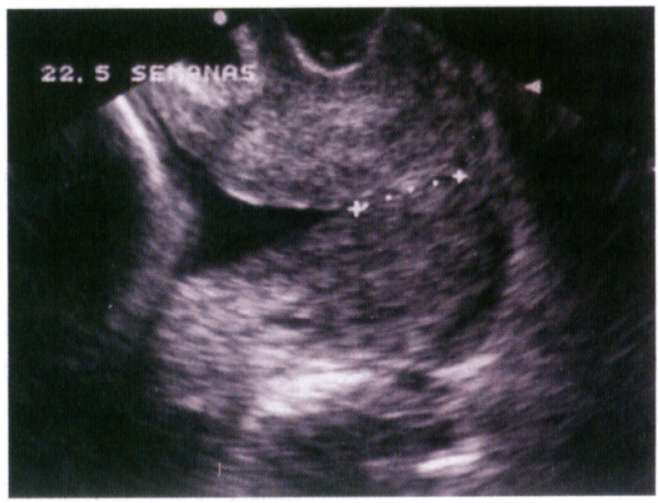

Figura 2 - Exame ultra-sonográfico evidenciando colo uterino em corte longitudinal. Observam-se o sinal do "afunilamento" e comprimento do colo reduzido. Não foi evidenciado o EGE. 
Este estudo não teve como objetivo o acompanhamento clínico pré-natal das mulheres envolvidas na pesquisa. Os dados relacionados à data do parto, via de parto e condições do recém-nascido foram obtidos por meio de telefonemas para cada uma das gestantes, aproximadamente 20 semanas após a realização do exame US. As pacientes não localizadas por esta via foram contactadas por carta explicativa previamente selada a qual questionava os mesmos dados anteriormente citados.

As variáveis qualitativas foram representadas por freqüência absoluta e relativa, e as variáveis quantitativas, por média, desvio padrão, mediana e valores mínimo e máximo. A associação entre as variáveis qualitativas foi avaliada pelo teste $\chi^{2}$ ou teste exato de Fisher. Para cada variável estudada, foi calculado o risco relativo seguido do intervalo com $95 \%$ de confiança. A técnica de análise de regressão logística univariada foi utilizada para verificar, entre as variáveis estudadas, quais foram indicativas para o PPT espontâneo. O nivel de significância adotado foi de 95\% (alfa = $5 \%$ ) e descritivos (p) iguais ou inferiores a 0,05 foram considerados significantes.

\section{Resultados}

Das 361 gestantes avaliadas, 18 (5,0\%) apresentaram PPT espontâneo em idades gestacionais que variaram entre 27 e 36 semanas. Destas, 11 $(3,0 \%)$ tiveram PPT antes da $36^{\mathrm{a}}$ semana, $8(2,2 \%)$ antes da $35^{\mathrm{a}}$ semana, $5(1,4 \%)$ antes da $34^{\mathrm{a}}$ semana e duas pacientes $(0,6 \%)$ em idade gestacional inferior à $33^{\mathrm{a}}$ semana. Quanto ao tipo de parto, 159 pacientes $(44,0 \%)$ tiveram parto cesáreo, 88 (24,4\%), parto normal espontâneo e $114(31,5 \%)$, parto normal induzido.

Ao utilizar-se para comprimento do colo um valor igual ou inferior a $20 \mathrm{~mm}$, como descrito na literatura como um dos limites mais empregados na predição de PPT espontâneo, pode-se verificar que ocorreu em $12(3,3 \%)$ gestantes na amostra total de 361 pacientes. Dentre as 18 gestantes que evoluíram para PPT espontâneo, 5 (27,8\%) apresentavam o comprimento cervical inferior a $20 \mathrm{~mm}$. Na Tabela 1 encontram-se descritos os valores de sensibilidade, especificidade, valor preditivo positivo e valor preditivo negativo do parâmetro "menor medida do comprimento cervical" menor ou igual a $20 \mathrm{~mm}$ na predição do PPT espontâneo nas diferentes idades gestacionais. A análise univariada revelou associação significativa entre o comprimento cervical igual ou inferior a 20 mm e PPT espontâneo (Tabela 2).

Tabela 1 - Medida do comprimento cervical inferior ou igual a $20 \mathrm{~mm}$ e associação com parto pré-termo espontâneo.

\begin{tabular}{|c|c|c|c|c|c|c|}
\hline \multirow{3}{*}{$\begin{array}{l}\text { Medida do } \\
\text { comprimento do } \\
\text { colo } \leq 20 \mathrm{~mm}\end{array}$} & \multicolumn{6}{|c|}{ IG ao nascimento (semanas) } \\
\hline & \multicolumn{2}{|c|}{$<37$} & \multicolumn{2}{|c|}{$<36$} & \multicolumn{2}{|c|}{$<35$} \\
\hline & $\mathbf{n}$ & $\%$ & $\mathrm{n}$ & $\%$ & $\mathrm{n}$ & $\%$ \\
\hline Sensibilidade & $5 / 18$ & 27,8 & $3 / 11$ & 27,3 & $3 / 8$ & 37,5 \\
\hline Especificidade & $336 / 343$ & 98,0 & $341 / 350$ & 97,4 & $344 / 353$ & 97,5 \\
\hline VPP & $5 / 12$ & 41,7 & $3 / 12$ & 25,0 & $3 / 12$ & 25,0 \\
\hline VPN & $336 / 349$ & 96,3 & $341 / 349$ & 97,7 & $344 / 349$ & 98,6 \\
\hline
\end{tabular}

IG = idade gestacional; VPP = valor preditivo positivo; VPN = valor preditivo negativo.

Tabela 2 - Distribuição de casos com comprimento do colo menor ou igual a $20 \mathrm{~mm}$ e ausência do EGE na amostra total e nas gestantes que tiveram parto pré-termo espontâneo e a termo.

\begin{tabular}{|c|c|c|c|c|c|c|c|c|c|}
\hline \multirow[t]{2}{*}{$\begin{array}{l}\text { Parâmetro } \\
\text { ultra-sonográfico }\end{array}$} & \multicolumn{3}{|c|}{$\begin{array}{l}\text { Amostra total } \\
\quad(n=361)\end{array}$} & \multicolumn{2}{|c|}{$\begin{array}{c}\text { IG ao nascimento } \\
<37 \text { semanas }(n=18)\end{array}$} & \multicolumn{2}{|c|}{$\begin{array}{c}\text { IG ao nascimento } \\
\geq 37 \text { semanas }(n=343)\end{array}$} & \multirow[t]{2}{*}{$\mathrm{p}$} & \multirow[t]{2}{*}{$\begin{array}{c}\text { RR } \\
\text { [IC 95\%] }\end{array}$} \\
\hline & & $\mathrm{n}$ & $\%$ & $\mathrm{n}$ & $\%$ & $\mathrm{n}$ & $\%$ & & \\
\hline \multirow{2}{*}{$\begin{array}{l}\text { Menor medida retilínea do } \\
\text { comprimento do colo } \leq 20 \mathrm{~mm}\end{array}$} & Pre & 12 & 3,3 & 5 & 41,7 & 7 & 58,3 & $<0,001$ & 11,27 \\
\hline & Aus & 349 & 96,7 & 13 & 3,7 & 336 & 96,3 & & {$[4,79 ; 26,53]$} \\
\hline \multirow[t]{2}{*}{ Ausência do EGE } & Pre & 10 & 2,8 & 8 & 80,0 & 2 & 20,0 & $<0,001$ & 28,57 \\
\hline & Aus & 351 & 97,2 & 10 & 2,8 & 341 & 97,2 & & {$[14,40 ; 56,68]$} \\
\hline
\end{tabular}

A ausência do EGE foi constatada em $10(2,8 \%)$ das 361 pacientes. Dentre as 18 mulheres que tiveram PPT espontâneo, o EGE não foi observado em 8 , ou seja, em $44,4 \%$ das gestantes. A distribuição da ausência do EGE observado nas diferentes idades gestacionais encontra-se demonstrada na Tabela 3. A análise univariada da ausência do EGE evidenciou $\mathrm{p}<0,001$ na predição de parto pré-termo (Tabela 2). O estudo comparativo entre os dados expostos nas Tabelas 1 e 3 revela valores de sensi- bilidade elevados na associação entre EGE ausente e PPT espontâneo quando comparados ao comprimento cervical igual ou inferior a $20 \mathrm{~mm}$. A sensibilidade para os parto em idade gestacional anterior à $35^{\mathrm{a}}$ semana foi de $75 \%$ quando constatado EGE ausente, ao passo que para comprimento cervical inferior a $20 \mathrm{~mm}$ foi de apenas $37,5 \%$. A mesma constatação é observada quanto ao valor preditivo positivo nos casos de PPT: dentre as 10 pacientes em que o EGE encontrava-se ausente, 6 
apresentaram partos anteriores à $35^{\mathrm{a}}$ semana. $\mathrm{O}$ comprimento do colo foi igual ou inferior a $20 \mathrm{~mm}$ em 12 pacientes e destas, apenas 3 evoluíram com parto anterior a 35 semanas.

Tabela 3 - Ausência de eco glandular endocervical (EGE) e associação com o parto prétermo espontâneo.

\begin{tabular}{|c|c|c|c|c|c|c|}
\hline \multirow[t]{3}{*}{ EGE ausente } & \multicolumn{6}{|c|}{ IG ao nascimento (semanas) } \\
\hline & \multicolumn{2}{|c|}{$<37$} & \multicolumn{2}{|c|}{$<36$} & \multicolumn{2}{|c|}{$<35$} \\
\hline & $\mathrm{n}$ & $\%$ & $n$ & $\%$ & $\mathrm{n}$ & $\%$ \\
\hline Sensibilidade & $8 / 18$ & 44,4 & $7 / 11$ & 63,6 & $6 / 8$ & 75,0 \\
\hline Especificidade & $341 / 343$ & 99,4 & $347 / 350$ & 99,1 & $349 / 353$ & 98,9 \\
\hline VPP & $8 / 10$ & 80,0 & $7 / 10$ & 70,0 & $6 / 10$ & 60,0 \\
\hline VPN & $341 / 351$ & 97,2 & $347 / 351$ & 98,9 & $349 / 351$ & 99,4 \\
\hline
\end{tabular}

IG = idade gestacional; VPP = valor preditivo positivo; VPN = valor preditivo negativo.

Detectou-se também associação significante entre os dois parâmetros avaliados e a ocorrência de PPT espontâneo $(\mathrm{p}<0,001)$, indicando que a presença de alteração nos parâmetros US é significantemente maior no grupo de pacientes que evolui para PPT quando comparado ao grupo de mulheres com parto a termo; contudo, embora com amplos intervalos de confiança, o risco relativo diante do EGE ausente mostrou-se mais elevado quando comparado ao risco relativo do comprimento do colo inferior a $20 \mathrm{~mm}$.

Foi observada associação significante entre EGE ausente e comprimento do colo inferior a 20 $\mathrm{mm}(\mathrm{p}<0,001)$. A presença de colo curto foi significantemente maior no grupo com ausência do EGE quando comparado ao grupo com presença do EGE.

Em sete pacientes verificou-se comprimento cervical igual ou inferior a $20 \mathrm{~mm}$ e EGE ausente; destas, apenas uma $(5,6 \%)$ apresentou PPT espontâneo. Em cinco pacientes o EGE encontrava-se ausente e o comprimento do colo era superior a $20 \mathrm{~mm}$, verificou-se que 4 destas pacientes parturiram em idades gestacionais anteriores à $37^{\mathrm{a}}$ semana.

\section{Discussão}

Foram estudadas mulheres grávidas da população geral. Ocorreram 18 PPT espontâneos na população de 361 pacientes, ou seja, em 5,0\% das pacientes estudadas. No Brasil, encontramos apenas as publicações de Carvalho et al. ${ }^{17}$ e FreitasJunior et al. ${ }^{21}$, que abordaram pacientes da população geral. Na UNIFESP-EPM há apenas uma publicação a respeito da avaliação US do colo durante a gravidez, que abordou exclusivamente pa- cientes de alto risco com antecedentes de $\mathrm{PPT}^{5}$. $\mathrm{Na}$ pesquisa da literatura pudemos observar apenas três publicações que descrevem a área das glândulas endocervicais na gravide $z^{18-20}$.

O exame US morfológico do segundo trimestre é preconizado no período de 18 a 24 semanas. Todavia, para avaliação minuciosa da anatomia cardíaca e de outros detalhes anatômicos, o periodo posterior à $21^{a}$ semana mostra-se mais adequado. Portanto, no período entre a $21^{\mathrm{a}}$ e a $24^{\mathrm{a}}$ semana, o médico pode verificar de forma mais acurada a anatomia fetal por meio do US morfológico e julgamos ser esta uma boa oportunidade de se avaliar o colo pela US-TV.

A sensibilidade e o valor preditivo positivo do comprimento cervical para indicação de risco para PPT são baixos na população geral, segundo relatos de várias publicações ${ }^{1,11,13}$. As maiores taxas de sensibilidade para predição de PPT por meio da medida do colo por ultra-sonografia são encontradas nos trabalhos que estudaram populações com algum outro fator de risco ${ }^{2,12}$, tais como antecedentes obstétricos de prematuridade. Tais indices variam também de acordo com a idade gestacional na qual são realizadas as medidas e com os critérios de avaliação do orifício cervical interno ${ }^{2}$. Com a utilização do comprimento cervical inferior ou igual a $20 \mathrm{~mm}$, observamos elevados indices de especificidade e valor preditivo negativo, ao passo que o valor preditivo positivo permanece baixo em todas as idades gestacionais. A sensibilidade, à semelhança dos relatos da literatura, apresenta-se baixa, com exceção para partos ocorridos anteriormente à $33^{a}$ semana. O reduzido número de partos observados neste período impede análise definitiva.

Carvalho et al. ${ }^{17}$ empregaram $20 \mathrm{~mm}$ como valor de corte em população geral da mesma cidade por nós analisada e obtiveram valores de sensibilidade de $42,3 \%$ e especificidade de $8,8 \%$ para predição do parto pré-termo. Estudos com grandes amostragens populacionais também têm revelado pontos de corte mais baixos do comprimento cervical como indicador de risco do PPT ${ }^{11,15}$. Em estudo colaborativo multicêntrico muito significativo, Iams et al. ${ }^{1}$, em 1998, obtiveram $25 \mathrm{~mm}$ como percentil 10 da curva populacional da medida do comprimento cervical entre a $24^{\mathrm{a}}$ e a $28^{\mathrm{a}}$ semana e com este ponto de corte obtiveram sensibilidade de $37 \%$ e especificidade de $92 \%$ para predição do PPT. Ao utilizaram o terceiro percentil, ou seja, $20 \mathrm{~mm}$, ocorreu redução da sensibilidade do método para $23 \%$ e elevação da especificidade para $97 \%$.

Neste estudo, modificamos o nome do sinal originalmente descrito como cervical gland area 
para EGE, pois julgamos o termo "eco" mais apropriado em relação à palavra "área" que significa, na lingua portuguesa, a medida de uma superficie. Utilizamos a expressão eco como um sinal US que indica uma faixa na imagem bidimensional, da mesma forma como é amplamente utilizado o termo "eco endometrial", que representa a região constituída pelo endométrio.

Os estudos anteriores que avaliaram tal marcador US o fizeram em idades gestacionais distintas. Sekiya et al. ${ }^{18}$, em 1998, observaram pacientes em estudo longitudinal no segundo e terceiro trimestres, entre 16 e 41 semanas. Yoshimatsu et al. ${ }^{19}$, em 2002, estudaram a quase totalidade das pacientes no terceiro trimestre, ao passo que Fukami et al. ${ }^{20}$ no corrente ano observaram pacientes entre a $16^{\mathrm{a}}$ e a $19^{\mathrm{a}}$ semana.

Observamos a ausência do EGE em 2,8\% das mulheres estudadas entre 21 e 24 semanas, ao passo que Sekiya et al. ${ }^{18}$ encontraram a endocervical gland area em todas as pacientes com idade gestacional inferior a 27 semanas. Fukami et al. ${ }^{20}$. verificaram a ausência do referido sinal em $0,36 \%$ da população avaliada entre 16 e 19 semanas, portanto em idade gestacional mais precoce quando comparada à população por nós estudada. A não-visualização do EGE apresenta freqüência crescente com o evoluir da gestação, como descrito inicialmente por Sekiya et al. ${ }^{18}$, porém com início mais precoce. Estudos futuros longitudinais poderão esclarecer de forma mais acurada este padrão no decorrer da gestação. No que tange à ausência da EGE nas pacientes que tiveram PPT, nossos dados apontam freqüência maior quando comparada aos dados disponiveis na literatura. Verificamos EGE ausente em 44,4\% das pacientes que evoluíram para PPT espontâneo, ao passo que Fukami et al. ${ }^{20}$ observaram-no em apenas $8,3 \%$ destas pacientes entre 16 e 19 semanas de gestação.

A ausência do EGE na predição do PPT apresentou valores de sensibilidade mais elevados no grupo das pacientes com PPT em idades gestacionais mais precoces. Acreditamos que a elevação do tamanho amostral possa revelar de forma mais evidente esta tendência, como evidenciou a publicação de Fukami et al. ${ }^{20}$. Os autores destacaram a elevada sensibilidade $(75 \%)$ da ausência do EGE na predição do PPT espontâneo em idade gestacional inferior a 32 semanas, ao passo que entre 32 e 36 semanas foi de apenas $2,3 \%$. Destacamos que o número de pacientes estudadas por Fukami et al. ${ }^{20}$ foi significativamente maior que o estudado por nós e provavelmente com o aumento da amostra poderemos observar diferenças mais significativas na capacidade de predição nas diferentes idades gestacionais do parto. Os indices de especificidade e valor preditivo negativo permaneceram elevados, em valores muito próximos a $100 \%$ em todas as idades gestacionais para predição de PPT espontâneo na ausência de EGE, em acordo com os resultados de Fukami et al. ${ }^{20}$. Tais dados indicam a reduzida probabilidade de PPT diante da visualização do EGE entre 21 e 24 semanas.

Observamos associação significante entre a ausência do EGE e o colo curto, indicando que diante de um colo com comprimento acima do ponto de corte, habitualmente o EGE é observado. Importante associação entre a detecção do EGE, comprimento cervical e idade gestacional no parto também foi observada por Yoshimatsu et al. ${ }^{19}$.

Dentre as pacientes estudadas, não foi detectado o EGE em 10. Destas, $80 \%$ tiveram parto em período anterior à $37^{\mathrm{a}}$ semana, traduzindo elevado valor preditivo positivo quando comparado à medida do comprimento cervical $(41,7 \%)$, o qual exibiu valores muito baixos nas diferentes idades gestacionais. A sensibilidade do encurtamento do colo (menor ou igual a $20 \mathrm{~mm}$ ) para predizer PPT espontâneo anterior à $37^{\mathrm{a}}$ semana também revelou-se mais reduzida $(27,8 \%)$ quando comparado à ausência do EGE. A não-visualização do EGE teve elevada sensibilidade e valor preditivo positivo quando comparada ao encurtamento do comprimento cervical nos resultados de Fukami et al. ${ }^{20}$.

$\mathrm{Na}$ avaliação conjunta do comprimento do colo e a presença ou ausência do EGE, das sete $(1,9 \%)$ pacientes que apresentavam comprimento do colo uterino menor ou igual a $20 \mathrm{~mm}$ e presença do EGE, seis parturiram a termo. De acordo com o exposto, a visualização do EGE proporciona, aparentemente, atenuação do risco de PPT diante do encurtamento do colo.

A análise interpretativa do risco relativo obtido nas duas variáveis apontam a ausência do EGE como o sinal com risco relativo mais elevado $(28,57)$, seguido da menor medida do colo menor ou igual a $20 \mathrm{~mm}(11,27)$. Estes dados indicam que o risco de PPT no grupo com ausência do EGE é 28 vezes maior que o risco de PPT no grupo com presença do EGE.

Entretanto, faz-se necessária a identificação dos fatores que levam ao desaparecimento do EGE de tal forma a compreendê-lo como parâmetro para a predição do PPT.

Quando o processo de amadurecimento cervical ocorre precocemente na gestação, freqüentemente leva ao PPT espontâneo. Por conseguinte, estratégia lógica para predizer o PPT espontâneo inicia-se com a identificação do amadurecimento cervical precoce. Mas qual seria o 
motivo do desaparecimento da imagem US correspondente às glândulas endocervicais (EGE) como marcador precoce do amadurecimento cervical? Uma das possibilidades é que as modificações bioquímicas presentes no colo em processo de amadurecimento, secundárias ou não a processo infeccioso, promovam modificações arquiteturais importantes em decorrência da intensa colagenólise, o que poderia promover o colapso da estrutura das glândulas endocervicais, com aplainamento das mesmas, de forma concomitante ou precedente ao apagamento do colo. Outra hipótese que poderia colaborar com o desparecimento do EGE consiste no acúmulo de água, o qual poderia promover redução significativa da ecogenicidade do estroma e, portanto, dificultaria a visualização da faixa do EGE. Estudos histológicos específicos do epitélio endocervical nas pacientes que apresentaram desaparecimento do EGE e/ou desenvolveram PPT espontâneo poderão auxiliar na elucidação destas hipóteses.

Provavelmente, com estudo multicêntrico colaborativo envolvendo numero maior de pacientes, realizado por profissionais capacitados para mensuração e análise morfológica cervical cuidadosa, seja possivel melhorar a predição das variáveis para o PPT espontâneo.

Os resultados desta pesquisa indicam que a ausência do EGE ao exame US durante o segundo trimestre constitui fator indicativo de PPT espontâneo a ser considerado, bem como o comprimento cervical, ao menos na população geral de gestações únicas. Este estudo revela tendência clara da marcante importância do EGE ausente como indicador do risco para o PPT, a ser confirmada em pesquisas futuras.

\section{ABSTRACT}

Purpose: to verify the prevalence of two sonographic findings, the cervical gland area (CGA) feature and the cervical length of less than $20 \mathrm{~mm}$, and to compare these with the risk for premature delivery in pregnant women between 21 and 24 weeks' gestation.

Method: this was a prospective, cross-sectional study in which 361 women were consecutively examined by transvaginal ultrasonography. Müllerian or other malformations, multiple gestations, fetal death, olygo- or polyhydramnios, marginal placenta previa, and conization, cerclage, amputation or other surgical procedures in the cervix, prior to or during pregnancy, were exclusion criteria. After the abdominal ultrasonographic morphological examination, we used transvaginal ultrasonography to measure the cervical length and to observe the presence of hyper-or hypoechoic area next to the endocervical canal, a feature characteristic of endocervical epithelium glands which is called CGA (cervical gland area). Qualitative variables are expressed as absolute and relative frequency. Quantitative variables are expressed as mean, median, standard deviation, minimum, and maximum values. Association between qualitative variables was detected by the $\chi^{2}$ test or by the Fisher exact test. For each variable, the relative risk and the 95\% confidence interval (CI) were calculated. Logistic regression analysis was used to calculate the predictive values for premature delivery. Significance level was 95\% (alpha $=5 \%)$, with descriptive $(p)$ values equal or lower than 0.05 considered significant.

Results: spontaneous preterm delivery occurred in $5.0 \%$ of the patients. Cervical length was up to $20 \mathrm{~mm}$ in $3.3 \%$ of all studied patients and in $27.8 \%$ of those who delivered spontaneously before the end of the pregnancy. Absence of the CGA was detected in $2.8 \%$ of all patients and in $44.4 \%$ of the women who eventually developed spontaneous preterm labor. There was a statistically significant association of absence of CGA with short cervical length ( $p<0.001)$. Absence of CGA was strongly associated with spontaneous preterm delivery (relative risk of 28.57, 95\% CI 14.40-56.68). Conclusion: the absent CGA feature is a new morphological ultrasonographic parameter that is useful in the prediction of spontaneous preterm delivery in single gestations. Our results show that the parameter can be used as an indicator of risk for premature delivery, to be confirmed by future research.

KEYWORDS: Cervix incompetence. Cervix uteri. Premature labor. Ultrasonography. Ultrasonics.

\section{Referências}

1. Iams JD, Goldenberg RL, Mercer BM, et al. The Preterm Prediction Study: recurrence risk of spontaneous preterm birth. National Institute of Child Health and Human Development MaternalFetal Medicine Units Network. Am J Obstet Gynecol 1998; 178:1035-40.

2. Guzman ER, Mellon C, Vintzileos AM, Ananth CV, Walters C, Gipson K. Longitudinal assessment of endocervical canal length between 15 and 24 weeks' gestation in women at risk for pregnancy loss or preterm birth. Obstet Gynecol 1998; 92:31-7.

3. Berkowitz GS, Papiernik E. Epidemiology of preterm birth. Epidemiol Rev 1993; 15:414-43.

4. Bittar RE, Yamasaki AA, Sasaki S, Zugaib M. Cervical fetal fibronectin in patients at increased risk for preterm delivery. Am J Obstet Gynecol 1996; 175:178-81.

5. Oliveira TA, Mariani Neto C, Siqueira AC, et al. Fibronectina fetal como fator de predição do parto prematuro em gestações de alto risco. Rev Bras Ginecol Obstet 1997; 19:305-11. 
6. Bittar RE, Yamasaki AA, Sasaki S, Galletta MA, Zugaib M. Determinação do risco para o parto prematuro através da detecção da fibronectina fetal na secreção cérvico-vaginal e da monitorização das contrações uterinas. Rev Bras Ginecol Obstet 1996; 18:165-72.

7. Rozenberg P, Gillet A, Ville Y. Transvaginal sonographic examination of the cervix in asymptomatic pregnant women: review of the literature. Ultrasound Obstet Gynecol 2002; 19:302-11.

8. Andersen HF, Nugent CE, Wanty SD, Hayashi RH. Prediction of risk for preterm delivery by ultrasonographic measurement of cervical length. Am J Obstet Gynecol 1990; 163:859-67.

9. Murakawa H, Utumi T, Hasegawa I, Tanaka K, Fuzimori R. Evaluation of threatened preterm delivery by transvaginal ultrasonographic measurement of cervical length. Obstet Gynecol 1993; 82:829-32.

10.Iams JD, Goldenberg RL, Meis PJ, et al. The length of the cervix and the risk of spontaneous premature delivery. National Institute of Child Health and Human Development Maternal Fetal Medicine Unit Network. N Engl J Med 1996; 334:567-72.

11. Heath VC, Southall TR, Souka AP, Elisseou A, Nicolaides KH. Cervical length at 23 weeks of gestation: prediction of spontaneous preterm delivery. Ultrasound Obstet Gynecol 1998; 12:312-7.

12.Berghella V, Tolosa JE, Kuhlman K, Weiner S, Bolognese RJ, Wapner RJ. Cervical ultrasonography compared with manual examination as a predictor of preterm delivery. Am J Obstet Gynecol 1997; 177:723-30.

13.Hassan SS, Romero R, Berry SM, et al. Patients with an ultrasonographic cervical length $<$ or $=15 \mathrm{~mm}$ have nearly a $50 \%$ risk of early spontaneous preterm delivery. Am J Obstet Gynecol 2000; 182:1458-67.

14.Guzman ER, Walters C, Ananth CV, et al. A comparison of sonographic cervical parameters in predicting spontaneous preterm birth in high-risk singleton gestations. Ultrasound Obstet Gynecol $2001 ; 18: 204-10$
15.To MS, Skentou C, Liao AW, Cacho A, Nicolaides $\mathrm{KH}$. Cervical length and funneling at 23 weeks of gestation in the prediction of spontaneous early preterm delivery. Ultrasound Obstet Gynecol 2001; 18:200-3.

16.Yamasaki AA, Bittar RE, Fonseca ESB, Martinelli S, Sasaki S, Zugaib M. Prevenção de parto prematuro: emprego do toque vaginal e da ultra-sonografia transvaginal. Rev Bras Ginecol Obstet 1998; 20:3506. 18 .

17. Carvalho MH, Bittar RE, Brizot ML, Maganha PP, Fonseca ESB, Zugaib M. Cervical length at 11-14 weeks' and 22-24 weeks' gestation evaluated by transvaginal sonography, and gestational age at delivery. Ultrasound Obstet Gynecol 2003; 21:135-9.

18.Sekiya T, Ishihara K, Yoshimatsu K, Fukami T, Kikuchi S, Araki T. Detection rate of the cervical gland area during pregnancy by transvaginal sonography in the assessment of cervical maturation. Ultrasound Obstet Gynecol 1998; 12:328-33.

19.Yoshimatsu K, Sekiya T, Ishihara K, Fukami T, Otabe $\mathrm{T}$, Araki T. Detection of cervical gland area in threatened preterm labor using transvaginal sonography in the assessment of cervical maturation and the outcome of pregnancy. Gynecol Obstet Invest 2002; 53:149-56.

20.Fukami T, Ishihara, K, Sekiya T, Araki T. Is transvaginal ultrasonography at mid-trimestrer useful for predicting early spontaneous preterm birth? J Nippon Med Sch 2003; 70:135-40.

21.Freitas-Júnior RAO, Maud-Filho F, Duarte G, Ferreira AC, Freitas AKMSO, Azevedo GD. Evolução do comprimento cervical uterino na gestação, avaliado pela ultra-sonografia transvaginal. Rev Bras Ginecol Obstet 2003; 25:115-21.

Recebido em: 25/11/2003 Aceito com modificações em: 1/3/2004

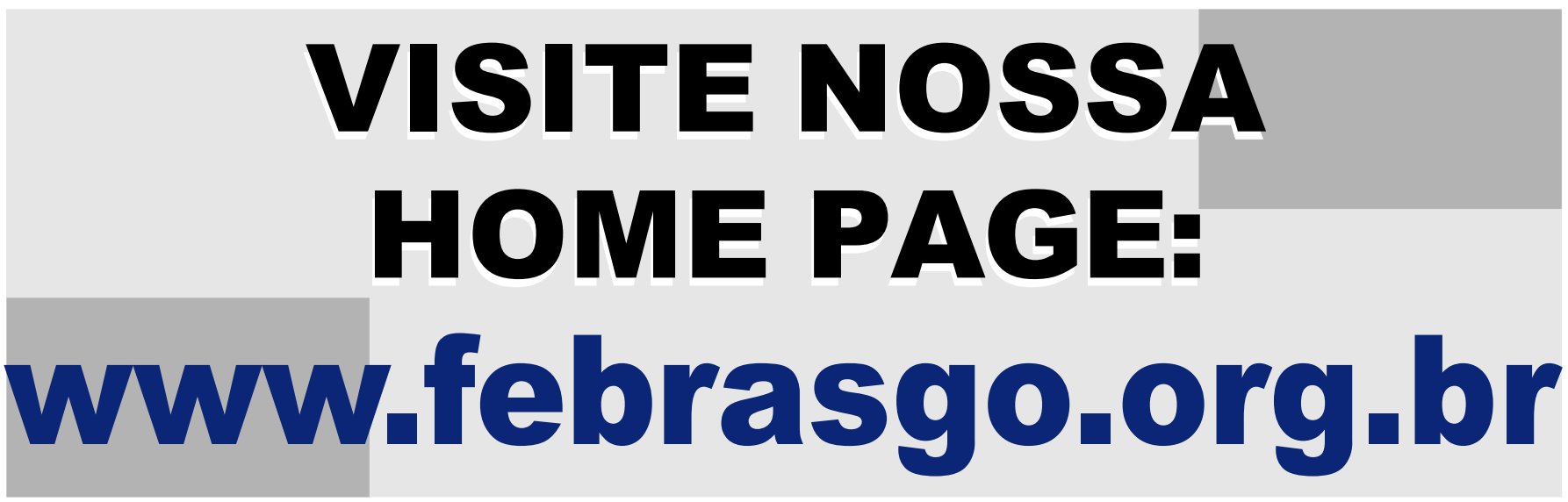

